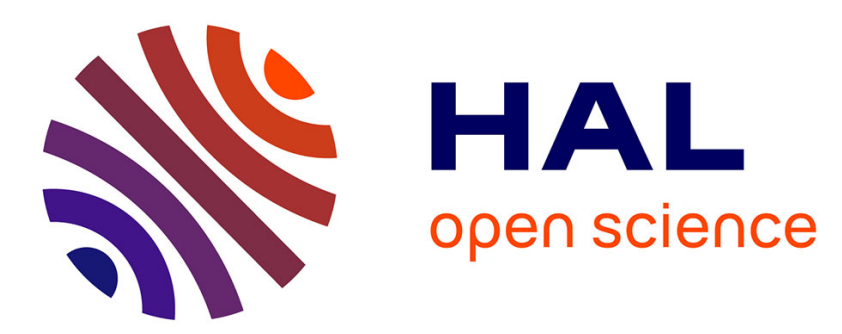

\title{
Vers une diplomatie des peuples? L'opinion publique et les crises franco-anglaises des années 1840 Renaud Meltz
}

\section{To cite this version:}

Renaud Meltz. Vers une diplomatie des peuples? L'opinion publique et les crises franco-anglaises des années 1840. Histoire, économie et société, 2014, 33 (2), p.58-78. 10.3917/hes.142.0058 . hal01777630

\section{HAL Id: hal-01777630 \\ https://hal.science/hal-01777630}

Submitted on 25 Apr 2018

HAL is a multi-disciplinary open access archive for the deposit and dissemination of scientific research documents, whether they are published or not. The documents may come from teaching and research institutions in France or abroad, or from public or private research centers.
L'archive ouverte pluridisciplinaire HAL, est destinée au dépôt et à la diffusion de documents scientifiques de niveau recherche, publiés ou non, émanant des établissements d'enseignement et de recherche français ou étrangers, des laboratoires publics ou privés. 


\section{VERS UNE DIPLOMATIE DES PEUPLES ? L'OPINION PUBLIQUE ET LES CRISES FRANCO-ANGLAISES DES ANNÉES 1840}

Renaud Meltz

Armand Colin | «Histoire, économie \& société »

2014/2 33e année | pages 58 à 78

ISSN 0752-5702

ISBN 9782200929336

Article disponible en ligne à l'adresse :

https://www.cairn.info/revue-histoire-economie-et-societe-2014-2-page-58.htm

\section{Pour citer cet article :}

Renaud Meltz, «Vers une diplomatie des peuples ? L'opinion publique et les crises franco-anglaises des années 1840 », Histoire, économie \& société 2014/2 (33e année), p. 58-78.

DOI 10.3917/hes.142.0058

Distribution électronique Cairn.info pour Armand Colin.

(C) Armand Colin. Tous droits réservés pour tous pays.

La reproduction ou représentation de cet article, notamment par photocopie, n'est autorisée que dans les limites des conditions générales d'utilisation du site ou, le cas échéant, des conditions générales de la licence souscrite par votre établissement. Toute autre reproduction ou représentation, en tout ou partie, sous quelque forme et de quelque manière que ce soit, est interdite sauf accord préalable et écrit de l'éditeur, en dehors des cas prévus par la législation en vigueur en France. Il est précisé que son stockage dans une base de données est également interdit. 


\title{
Vers une diplomatie des peuples? L'opinion publique et les crises franco-anglaises des années 1840
}

\author{
Par Renaud Meltz
}

\begin{abstract}
Résumé
Un premier système médiatique s'organise à partir des années 1830, en Europe occidentale, autour de la presse écrite. La politique étrangère y tient une place primordiale. L'opinion, nouvelle actrice des relations internationales, revendique la publicité de la diplomatie et s'érige en instance de jugement. Cette opinion se fait entendre, sous différentes formes sociales, pour réclamer une politique étrangère morale, face à un État libéral qui se cantonne à la sphère de ses intérêts. Cette exigence d'une politique bonne et, partant, universelle, favorise l'émergence d'une opinion publique internationale, partageant des valeurs. Naturellement, cette OPI demeure à la mesure de l'étroit espace public européen. Le propos de cet article est de montrer que, contre le sentiment et les discours des décideurs, l'affirmation de l'opinion publique dans les affaires internationales a produit des effets principalement iréniques. La meilleure connaissance réciproque a permis d'apprivoiser la culture médiatique adverse serait-ce dans l'affrontement verbal. En défendent la paix entre nations civilisées d'égale dignité, contre le bellicisme d'État, les opinions publiques françaises et anglaises ont même participé à l'invention de la notion d'arbitrage : le tribunal informel de l'opinion a préfiguré, en somme, la judiciarisation de la société internationale. L'opinion publique a également revendiqué une démocratisation de chaque État, et de leurs relations, au bénéfice de la paix. L'appel à une diplomatie des peuples, affilié aux projets de paix hérités de Kant, dépassait-il la notion d'opinion en proclamant le refus des peuples d'être opposés entre eux, posture consubstantielle au refus d'être privé, pour chacun d'entre eux, de la maîtrise des questions de politique étrangère.
\end{abstract}

\section{Abstract}

A first media system gets organized from the 1930's, in the Western Europe, around the written press. The foreign policy has an essential place in it, allowing the public opinion to seize international affairs. This new actor of the international relations claims the advertising of the foreign policy and establish itself as a judgment authority; indeed, it considers itself the main moral resource of the country and its power's founding principle. This opinion makes it heard to claim a moral foreign policy, in front of a liberal state whose main interests are its own. This demand of a good and universal policy promotes the emergence of an international public opinion, sharing values. This initiation stays in the tight European public space. The aim of this article is to show that, against 
the decision-maker's feeling, the assertion of the public opinion in international affairs has mainly generated peace effects. The best mutual knowledge has allowed taming the opposite media culture. Fighting for peace between civilized nations of equal dignity, against the State warmongering, the French and English public opinion have participated to the invention of the mediation concept : as a matter of fact, the informal tribunal of the opinion prefigured the judiciarization of the international society. The public opinion has also claimed a democratization of each state, and of their relations, for peace. In this way, the call to a diplomacy of the peoples, affiliated to the peace project inherited from Kant, has been exceeding the notion of an opinion proclaiming the refusal of the people to be opposed between them, consubstantial place to the refusal to be private, for all of them, of the command of the foreign policy questions.

L'opinion publique existait-elle dans le premier $\mathrm{XIX}^{\mathrm{e}}$ siècle, si elle a jamais existé, pour reprendre le titre provocateur de Pierre Bourdieu ? Les historiens des monarchies constitutionnelles n'en doutent pas, qui l'identifient naturellement comme une actrice des relations internationales. Sans discuter sa nature, ni les mécanismes de son influence, ils admettent son rôle dans les crises franco-anglaises qui agitèrent les années 1840.

C'est vrai pour la crise d'Orient, dont le règlement, au détriment du pacha d'Égypte, Mehemet-Ali fut un camouflet pour la France, sa protectrice. Sa mise à l'écart du traité signé à Londres le 15 juillet 1840 par les grandes puissances ouvrit une longue période de tension entre les gouvernements français et anglais, attisée par leurs opinions respectives. Le vicomte de Guichen, dans sa monographie sur la question orientale, en 1921, opposait la sagesse du gouvernement français à la déraison d' " une opinion publique favorable à une cause chevaleresque ${ }^{1} \gg$. L'affaire Pritchard, épisode peu étudié et mal connu de la rivalité qui opposait les marins et les missionnaires de France et d'Angleterre, n'a pas moins mobilisé l'opinion publique des deux côtés de la Manche. Les Britanniques s'enflammèrent massivement en faveur du missionnaire et consul Pritchard, expulsé de Tahiti par les marins français, qui l'accusaient de menées séditieuses. Les Français s'indignèrent presque unanimement des paroles du premier ministre anglais, qui condamna ce " grossier outrage » à la Chambre des Communes, et réclama des « réparations ${ }^{2}$ ».

Dans cette époque immédiatement antérieure à la naissance d'une culture de masse, ces crises montrent que l'opinion publique ne relevait pas d'une simple catégorie discursive, agitée par le pouvoir et les médias. L'objet de cet article est d'identifier sa réalité sociale, dans chacun de ces pays, et sa capacité d'agir en se saisissant des questions de politique étrangère (ce que les politistes appellent l'opinion publique sur l'international, ou OPSI). Les crises franco-anglaises des années 1840 sont également l'occasion d'observer l'émergence d'une opinion publique internationale (OPI). Cette OPI devient possible quand le développement de la presse dilate l'espace public transnational au-delà du cercle de l'ancienne République des lettres, qui ne mobilisait qu'une étroite élite européenne en faveur de causes communes comme l'indépendance de la Grèce ${ }^{3}$.

Au cours des crises qui agitent les relations franco-anglaises dans les années 1840, l'OPSI et l'OPI s'introduisent comme de nouvelles actrices des relations internationales, portant une exigence inédite de pratiques diplomatiques plus transparentes et moralisées.

1. Vicomte de Guichen, La crise d'Orient de 1839 à 1841 et l'Europe, Émile-Paul, 1921, p. VII.

2. Voir Philippe Darriulat, «L'affaire Pritchard, un paroxysme de l'anglophobie française ? », Sylvie Aprile, Fabrice Bensimon (dir.), La France et l'Angleterre au XIX ${ }^{e}$ siècle : Échanges, représentations, comparaisons, Paris, Créaphis, 2006, p. 219-35 et Renaud Meltz, « L'affaire Pritchard », Revue d'histoire diplomatique, 2012.

3. Hervé Mazurel, Vertiges de la guerre. Byron, les philhellènes et le mirage grec, Les Belles Lettres, 2013. 
Encore faut-il s'entendre sur les termes. L'opinion fut-elle une instance de jugement moral ? La réponse est assurément positive : avant même de promouvoir les valeurs qu'elle ne trouvait pas assez bien défendues par l'État, l'opinion domestique, par sa seule identification à une sorte de tribunal collectif, incarnait la force morale d'un pays et la garantie de sa moralité, face à un État libéral qui se cantonnait à la sphère de ses intérêts.

En défendant une diplomatie plus juste que celle du gouvernement, en partageant des valeurs de peuple à peuple, les opinions française et anglaise des années 1840 ont contribué à l'émergence timide d'une opinion publique internationale, susceptible d'apparaître comme un recours à l'injustice par un arbitrage sinon juridique, au moins moral.

Mais l'espace public européen était trop peu abouti, dans le premier XIX ${ }^{\mathrm{e}}$ siècle, pour que la notion d'arbitrage soit formalisée par des institutions capables d'exercer juridiquement l'exigence commune de justice dans les rapports internationaux. Une autre voie s'esquissait, qui répondait à l'idéal kantien d'une fédération d'États libres : le refus, par les peuples, de se laisser confiner à la liberté d'opiner dans l'espace public abstrait de discussions tranchées par l'étroit pays légal ; la volonté de se ressaisir, comme un peuple agissant et souverain, dans le lieu réel du pouvoir, pouvoir de décider une politique juste. La faible postérité immédiate de cet idéal ne justifie pas son effacement historiographique.

\section{L'opinion publique, nouvelle actrice des relations entre les monarchies constitutionnelles}

L'opinion publique existe dans les monarchies constitutionnelles, serait-ce comme réalité discursive. Le discours sur l'opinion y était tenu par au moins trois institutions, qui fondaient leur légitimité sur elle ou prétendaient la dire et la faire : l'exécutif, le législatif et la presse.

En ce qui concerne le discours de l'État sur l'opinion publique, les conceptions semblent contradictoires, à première vue, entre les historiens et les politistes. Les premiers déduisent généralement la méfiance de l'État à l'égard de l'opinion publique de la pratique du contrôle des esprits. Les seconds considèrent que l'État a favorisé l'avènement d'une opinion publique pour mieux expulser le peuple de la place publique. Pour le politiste Dominique Reynié, qui se fonde sur des sources normatives, depuis la Révolution, l'État a favorisé la liberté d'opiner plutôt que celle de s'associer, et la discussion dans un espace public abstrait, plutôt que la prise de parole sur la place publique ${ }^{4}$. Conséquent, l'exécutif a cherché à éclairer l'opinion qu'il promouvait pour se légitimer à moindres frais. L'opinion publique, c'était la représentation et l'expression de la société civile, cette société qui fondait l'autorité de l'État sans se confondre avec lui ni même adhérer à ses décisions : « quand le public est libre, il faut absolument qu'il comprenne » résumait Guizot, théoricien puis acteur de cette histoire ${ }^{5}$.

L'opinion publique, permise par la distinction entre la société civile et l'État, théorisée par Benjamin Constant sous la Restauration, ne constituait pas seulement l'outil de légitimation de l'État libéral le moins périlleux, de préférence au suffrage universel ou quelque autre instrument attribuant le pouvoir au peuple ${ }^{6}$. En traitant des questions internationales,

4. Dominique Reynié, Le triomphe de l'opinion publique. L'espace public français $d u X V I^{e}$ au XXe siècle, Odile Jacob, 1998.

5. Servane Marzin, «L'Europe de François Guizot : le dernier échec ? », François Guizot, 1787-1874, L'Harmattan, 2010, p. 319-331.

6. Benjamin Constant, « De la liberté des anciens comparée à celle des modernes », 1819, Écrits politiques, Gallimard, 1997. Pour Constant, le bonheur de chacun et le bien de tous supposent que les citoyens exercent « leur droit de contrôle et de surveillance par la manifestation de leurs opinions », p. 619. 
elle offrait à l'État libéral une marge de négociation nouvelle avec ses partenaires étrangers, libéraux ou despotiques. Découvrant le traité de Londres, fin juillet 1840, en sa qualité d'ambassadeur de France en Angleterre, Guizot, pour en obtenir la révision, représenta le péril d'une opinion publique française mécontente jusqu' au bellicisme, après avoir identifié, comme historien, l'opinion publique comme le nouveau fondement de la puissance publique $^{7}$. Palmerston, avec mauvaise foi et habileté, renversa l'argument : il ne pouvait pas croire qu'un traité, mécontentant ponctuellement l'opinion, puisse être un motif de rupture entre deux pays qui n'étaient pas unis par le seul vœu de leurs gouvernements, mais par celui de leurs peuples ${ }^{8} \ldots$

Légitimé à moindre péril par une opinion publique qui lui permettait de mieux négocier avec ses partenaires, l'État libéral n'en tenait pas moins un discours de défiance sur l'opinion publique, fractionnée, irrationnelle, et partant immorale. Les historiens ont montré comment les pratiques de surveillance ont contribué à institutionnaliser l'opinion et à lui conférer un caractère réflexif ${ }^{9}$. Aussi bien, même les libéraux les moins inquiets d'élargir la base électorale du pays légal, comme Rémusat, dénonçaient la prétention belligène de l'opinion à discuter les questions de politique internationale :

Je suis forcé d'avouer que là où l'opinion publique entre au Conseil, que dans les pays libres, le danger d'être ainsi mené à ériger les petites affaires en grandes affaires et de s'engager dans l'action pour une parole, est une suite de la nécessité de tout discuter avec une publicité et une solennité égales ${ }^{10}$.

Bref, s'il faut faire advenir une opinion publique, il faut aussi la craindre, l'éclairer, et surtout lui résister, estime la monarchie constitutionnelle, par la voix de Guizot ${ }^{11}$.

Quant aux Chambres, à défaut d'être élues par l'ensemble du peuple, elles prétendaient dire l'opinion publique, y compris pour les questions internationales. Il est établi que les affaires étrangères suscitaient la plus grande animation des débats et la plus forte participation des députés ${ }^{12}$. Lorsque Guizot refusa, aux débuts de l'affaire Pritchard, de s'expliquer devant la Chambre des Pairs, le marquis de Boissy affirma solennellement que cette dernière était l'expression légale de l'opinion : «L'opinion publique s'est émue ; je demande qu'elle soit prise en considération. Messieurs, la chambre des pairs est seule à siéger dans ce moment; sa responsabilité n'en est que plus grande ${ }^{13}$. »

7. Archives du ministère des Affaires étrangères (AMAE), Correspondance politique, Angleterre, 655, f. 200'. Voir Pierre Manent, Histoire intellectuelle du libéralisme, Pluriel, 1997, p. 203, citant l'opuscule de 1822, De la peine de mort en matière politique : «La puissance a quitté les individus, les familles ; elle est sortie des foyers qu'elle habitait jadis ; elle s'est répandue dans la société tout entière ; elle y circule rapidement, à peine visible en chaque lieu, mais partout présente. Elle s'attache à des intérêts, à des idées, à des sentiments publics dont personne ne dispose, que personne même ne représente assez pleinement pour que leur sort dépende un moment du sien ».

8. AMAE, Correspondance politique, Angleterre, 655, f. 198', discussion relatée par Guizot à Thiers, le 25 juillet 1840 : «nos deux pays resteront unis [...] par les liens les plus puissants d'opinion, de sentiment, d'intérêt ».

9. Pierre Karila-Cohen, L'État des esprits. L'invention de l'enquête politique en France (1814-1848), PUR, 2008. L'enquête se limite aux questions domestiques.

10. Charles de Rémusat, Mémoires de ma vie, Plon, 5 vol., t. 4, 1962, p. 54,

11. Laurent Theis, François Guizot, Fayard, 2008, p. 394.

12. Louis Girard, William Serman, Edouard Cadet, Rémi Gossez, La chambre des Députés en 1837-1839, Sorbonne, 1976, p. 152.

13. Relaté par Le Globe, le 4 août 1844. 
La presse, enfin, prétendait dire et faire l'opinion publique, portant un discours sur sa propre légitimité à représenter l'opinion ${ }^{14}$. Face au déficit de la représentation électorale, la presse d'opposition prétendait constituer la chambre d'écho du peuple. Le National interprétant régulièrement la représentativité de ses idées, contre celles du pays légal, à partir de signes qui renvoient aux lieux publics, en fournissant la relation, par exemple, des banquets républicains, en pleine crise d'Orient ${ }^{15}$.

La presse gouvernementale, de son côté, espérait modeler le public, en l'informant de ses vues, sans se bercer d'illusion. Guizot, qui comptait sur le Journal des débats, pendant l'affaire Pritchard, admettait les limites de son influence : «Il m'échappe de temps en temps, tantôt par inadvertance, tantôt à dessein, pour faire acte d'indépendance ${ }^{16}$. » Mais Le Semeur, titre protestant, s'accordait avec la presse britannique pour penser qu'une idée, un parti, ne pesaient rien, dans les affaires publiques, sans le soutien d'un titre : " "Qu'est-ce qu'un parti politique ou religieux sans une revue ? Une cloche sans marteau qui se balance sans produire de son." Ainsi s'exprimait récemment l'un des plus spirituels collaborateurs de la Revue d'Édimbourg ${ }^{17}$. »

Sensible aux critiques sur sa représentativité, la presse délivrait un discours de justification et d'autocritique sur sa capacité à dire, malgré sa diversité, une opinion publique éclairée, selon elle, et capable d'unité. La presse d'opposition, au premier chef, répondait au reproche d'éclatement de l'opinion que, sur les questions de politique étrangère, il n'y a pas d'esprit de parti, mais une opinion unanime. Au lendemain du refus de Guizot d'annexer Tahiti, le Persévérant, de Limoges, exprima son vœu que l'OPSI fût durablement unifiée, fut-ce au détriment de l'émergence d'une opinion publique internationale : «Il n'y aurait plus que deux partis en France, celui des Français et celui de l'étranger ${ }^{18}$. » Pendant les crises qui agitent les relations franco-britanniques, la presse de chaque pays participe pourtant, de fait, à l'émergence d'une telle OPI, en invoquant régulièrement l'opinion publique du pays rival, sa presse et l'image produite sur la scène européenne. La presse anglaise se réfère quotidiennement aux « Paris papers » quand les journaux français évoquent symétriquement «les feuilles anglaises ». Quand la presse anglaise s'étonne, avec le Morning Chronicle, «l'organe semi-officiel de lord Palmerston » selon le National : «Les journaux de Paris, et le public parisien sont occupés par l'affaire de Tahiti, presque à l'exclusion de toute autre. Il est difficile de concevoir le brouhaha que causent la reine Pomare et ses États dans la capitale française ${ }^{19}$ »; la presse française ne s'émeut pas moins du bruit britannique : «La presse anglaise et les orateurs du parlement continuent à adresser à la France les plus grossières injures ${ }^{20}$. » Ces lectures croisées fonctionnent en écho et alimentent les représentations de soi et de l'autre, grâce une circulation déjà très fluide de l'information. La presse cite jusqu'aux titres les plus confidentiels du pays adverse, selon les attendus de l'argumentaire à déployer. The Standard du 27 février 1844, par exemple, cite Le Semeur, journal des protestants français, très concerné par l'affaire

14. Nous avons dépouillé systématiquement une quinzaine de titres anglais et autant de titres français pour cette période ; la numérisation de la presse anglaise permet de passer les articles dans le tamis très fin de la recherche par mots-clefs.

15. Le 2 septembre 1840 .

16. Archives nationales (AN), 42 AP 8, lettre de Guizot du 7 novembre 1844 à Bresson, cité par Servane Marzin, op. cit., p. 325.

17. Le Semeur, le 7 août 1844.

18. Cité dans la revue de presse du National du 11 mars 1844.

19. Le 29 février 1844.

20. Le National, le 4 août 1844. 
Pritchard, longtemps favorable au point de vue anglais, mais très peu diffusé en France même.

Le discours des journalistes sur l'opinion est naturellement adossé à un discours théorique. Les avancées les plus nettes, sur le plan d'un discours normatif, viennent d'outreManche. En 1839, la traduction française du Plan d'une paix universelle et perpétuelle que Jeremy Bentham avait écrit en 1789 met en discussion la nécessité d'une publicité des débats et des traités, en matière de relations internationales, pour fonder la paix, même s'il est difficile d'évaluer la pénétration de ces idées dans le public français. Le projet de Bentham, inspiré par les écrits de Kant, repose sur la création d'une Diète ou Tribunal de la paix, réunissant les représentants des États ; ses « opinions » prendraient force de loi par leur seule publicité, pourvu, précisait le penseur anglais, que la liberté de la presse fût garantie dans l'ensemble des États ${ }^{21}$.

Si l'opinion publique est une réalité discursive, il importe de l'identifier aussi comme une catégorie d'acteur, correspondant à une réalité sociale ayant sa propre capacité d'action politique, en dépit de son caractère insaisissable.

La réalité sociale et politique de l'opinion publique a suscité une abondante littérature depuis l'ouvrage fondateur d'Habermas, L'espace public. Sans trancher la controverse, relevons le nombre inédit de lecteurs d'une presse qui fait une large place aux questions internationales, en même temps que s'installe une culture médiatique inédite ${ }^{22}$.

La presse, sous la monarchie de Juillet, notamment au milieu des années 1840, connaît une croissance considérable du nombre de ses titres (230), de ses tirages (148000 exemplaires), de ses abonnés (presque 100 000), et de ses lecteurs, parmi la population alphabétisée - la moitié environ des Français -, grâce à des circuits peu visibles de diffusion : prêts en famille, cercles, cafés, et cabinets de lecture, dont les clients demeurent des bourgeois plutôt que des ouvriers, mais dont le nombre croît très sensiblement lors des crises internationales, selon la préfecture de police ${ }^{23}$. Pendant la crise d'Orient, la préfecture observe : «L'inquiétude est extrême dans Paris ; les cabinets de lecture sont remplis de personnes qui s'arrachent les journaux ${ }^{24}$. » Aux feuilles politiques, qui soutiennent une opinion, un parti, une personne, s'ajoute une presse commerciale (Le Siècle, La Presse), qui ouvre largement ses colonnes aux questions de politique étrangère. Le journal devient « le principal vecteur de la parole sociale », inaugurant un premier système médiatique ${ }^{25}$. Cette rupture culturelle permet la dilatation de l'espace public, une politisation accrue des masses et l'émergence d'une OPSI.

Le processus est globalement comparable en Grande-Bretagne : si le nombre de titres y est nettement moins important, cette concentration est le signe d'une plus grande maturité

21. Julien Cazala, « Jeremy Bentham et le droit international », Revue générale de droit international public, 2005, n 2, p. 363-387.

22. La bibliographie est désormais très touffue sur la question. Parmi les synthèses récentes : Christophe Charle, Le siècle de la presse, Seuil, 2004 et Dominique Kalifa, Philippe Régnier, Marie Ève Thérenty et Alain Vaillant (dir.), La civilisation du journal, histoire culturelle et littéraire de la presse française au XIXe siècle, Nouveau monde éditions, 2011. Sur la protohistoire de la culture médiatique en Europe occidentale, voir Alain Vaillant, «Invention littéraire et culture médiatique au XIX ${ }^{\mathrm{e}}$ siècle », Jean-Yves Mollier, Jean-François Sirinelli, François Vallotton (dir.), Culture de masse et culture médiatique en Europe et dans les Amériques, 1860-1940, PUF, 2006.

23. Françoise Parent-Lardeur, Les cabinets de lecture. La lecture publique à Paris sous la Restauration, Payot, 1982.

24. AN, F7 3890, rapports quotidiens du préfet de police au ministre de l'intérieur (Bulletins de Paris), le 6 octobre 1840 .

25. Alain Vaillant, «Invention littéraire et culture médiatique au XIX ${ }^{\mathrm{e}}$ siècle », op. cit., p. 20. 
économique et technique du secteur, sur un marché fort dynamique. On estime à quelque 270000 exemplaires la vente des journaux dominicaux, à la fin des années 1840, en GrandeBretagne, où l'alphabétisation, plus précoce que sur le continent, élargit encore le lectorat potentiel de la presse ${ }^{26}$.

Dans quelle mesure cette presse fabrique-t-elle, dans le double sens d'instituer et d'influencer, l'opinion publique ? Question considérable, à laquelle les organes de l'État monarchique apportent une réponse assurée : la presse est responsable de la politisation des masses et de leur intérêt pour les questions extérieures ; elle risque de provoquer le retour du public au peuple, de l'opinion à la manifestation. Au paroxysme de la crise d'Orient, la préfecture de Police s'inquiète, à Paris : «L'excitation des journaux, [rend] le moment difficile ; il ne sera pas extraordinaire si quelque démonstration populaire ou anarchique ne vient à se manifester ${ }^{27}$. »

La rumeur dessine un espace public plus large que celui formé par le cercle essentiellement urbain des lecteurs de la presse. Si les journaux hostiles au gouvernement, patriotes comme légitimistes, offrent un reflet probablement exagéré des sentiments belliqueux de l'ensemble du public pendant la crise d'Orient, le préfet de police donne à voir des signes d'émotion populaire dans le public qui ne lit pas nécessairement cette presse ; il offre à l'historien une précieuse revue des lieux où s'élabore cette rumeur publique, des salles de spectacles à la rue : «Hier, on a demandé à plusieurs reprises la Marseillaise dans tous les théâtres, et le parterre et le paradis de répéter l'air exécuté par l'orchestre ; ce soir encore on remarquait dans les rues de nombreux groupes d'ouvriers et d'étudiants chantant le même air $[\ldots]$ et tenant de mauvais $\operatorname{propos}^{28}$. »

Les Chambres, en dépit de leur imparfaite représentativité, contribuent à la formation d'un espace public ouvert aux affaires étrangères; on le vérifie par un phénomène de vases communicants. Pendant la crise d'Orient, la préfecture de police observe : « La convocation des chambres a calmé les esprits d'une manière sensible, et quelque grande que soit l'inquiétude qui règne sur les affaires du dehors, les agitateurs du dedans voient s'échapper par l'ordonnance publiée ce matin, un des prétextes dont ils s'armaient pour argumenter contre le pouvoir ${ }^{29}$. »

Il faut aussi compter, parmi les formes sociales que prend l'opinion publique, et la diversité de sa capacité d'expression, les manifestes, les bulletins de partis, les pétitions, les souscriptions populaires. La presse patriote lance une souscription, en 1844, pour offrir une épée d'honneur à l'amiral Dupetit-Thouars, dont Guizot a désavoué la décision d'annexer Tahiti. L'usage généralisé de la liberté d'opiner et de délibérer publiquement des affaires internationales se mesure également dans le meeting, qui prend une ampleur considérable en Angleterre, et le banquet, qui en tient lieu en France, « cette solennelle manifestation de l'opinion publique » selon la presse d'opposition. Ces formes sociales ne prétendent pas seulement incarner l'opinion publique, mais dicter l'agenda des affaires, pour mettre à l'ordre du jour de la grande discussion nationale un sujet escamoté ou mal évalué par le

26. Hannah Barker, Newspapers, Politics and English Society, 1695-1855, Pearson Education Limited, 2000, p. 33, citant Raymond Williams, London, 1961, p. 54. Voir aussi Fabrice Bensimon, « L'émergence d'une culture de masse en Grande-Bretagne, 1850-1914 », Culture de masse et culture médiatique, op. cit.

27. AN, F7 3890, le 3 octobre 1840.

28. Ibid. le 19 octobre 1840 . Le 5 octobre, déjà, la préfecture de police observe : « à l'opéra, entre deux actes, quelques personnes placées dans le parterre ont vivement demandé la Marseillaise, et sur le refus que faisait l'orchestre, se sont mis à la chanter ; aussitôt leur exemple a été suivi par la plus grande partie des spectateurs placés au parterre ».

29. Ibid., le 8 octobre. 
pouvoir $^{30}$. Il faut aussi compter les « petits imprimés » : libelles, tracts, recueils d'articles, et paroles de chansons ${ }^{31}$. Les fluctuations de la Bourse, enfin, fournissent un indice de l'évaluation collective de la probabilité de guerre ${ }^{32}$. Autant de signes qui ne permettent pas de douter de l'existence d'une OPSI, si l'on admet l'extravagance du singulier, étant entendue la diversité des formes de politisation et de saisie des questions internationales par les sociétés françaises et anglaises.

Cette OPSI incarne une sorte d'instance de jugement, et plaide pour une diplomatie qui ne soit pas seulement la défense d'intérêts, mais de valeurs; en cela, elle intéresse à double titre la notion de diplomatie morale.

\section{L'opinion, instance de jugement en faveur d'une diplomatie moralisée}

Dans la presse des années 1840, le mot « moral » est peu utilisé pour les affaires étrangères, avec une variété d'acceptions assez importante de surcroît. On distingue au moins trois usages de la notion :

- L'opinion revendique la publicité de la politique étrangère afin de s'ériger en instance de jugement moral.

- L'opinion publique est considérée comme la principale ressource morale du pays et partant le fondement de sa puissance.

- Troisième acception, enfin, la plus attendue, mais pas forcément la plus répandue : l'opinion exige une politique étrangère morale.

Pour qu'il existe une instance de jugement moral, encore faut-il qu'il y ait publicité des décisions. En ce sens, l'opinion publique apparaît comme une sorte de succédané de la souveraineté populaire pour l'opposition radicale, qui développe une morale de la publicité de la politique étrangère.

En attendant que la représentativité des Chambres soit améliorée, c'est l'opinion publique qui doit évaluer la justesse de la politique étrangère, estime la presse d'opposition au régime, pendant la crise d'Orient. Le bonapartiste Capitole, par exemple : « M. Thiers [a l'intention ]de convoquer la chambre pour s'en faire un appui dans les graves conjonctures qui se présentent. [...]. C'est à l'opinion publique seule que le ministère doit s'adresser ${ }^{33}$. » On trouve plus souvent l'expression de cette revendication en France qu'en Angleterre, où les journalistes et les débats au Parlement sont plus libres. Les acteurs les plus politisés de l'opinion française s'informent d'ailleurs de l'autre côté de la Manche, et font circuler les recueils de correspondance diplomatique qu'elle y trouve ${ }^{34}$.

30. Vincent Robert, Le temps des banquets. Politique et symbolique d'une génération (1818-1848), Publications de la Sorbonne, 2010. L'auteur ne fait pas de place aux affaires étrangères, dans son étude du mouvement pour la réforme électorale des années 1838-1841; elles occupent pourtant une large place dans les banquets. L'auteur déplore, à raison, (p. 243), que « le primat de la politique extérieure » ait occulté cette campagne nationale de banquets « dans les histoires de la monarchie de Juillet »; ce constat ne doit pas cacher en retour l'imbrication des questions extérieures et des revendications domestiques concernant le droit de vote.

31. Philippe Darriulat, La muse du peuple, chansons politiques et sociales en France 1815-1871, Presses universitaires de Rennes, 2010. Voir p. 30-31 pour les chansons anglophobes qui brocardent Guizot en général, et sa politique tahitienne en particulier.

32. Le 20 août 1840, le Morning-Chronicle rassure son lectorat en voyant dans la hausse de la bourse de Paris le signe que l'opinion française ne veut pas la guerre.

33. Le Capitole du 28 juillet 1840.

34. Correspondance relative to the affairs of the Levant, presented to both houses of Parliament by command of the Majesty, Harrison, London, 1841. 
L'exigence de publicité, comme fondement d'une politique étrangère juste, trouve des satisfactions dans l'émergence d'un espace publique européen. Le Constitutionnel du 8 septembre 1844, qui déplore le défaut de transparence du gouvernement français, observe que le public français s'informe par le détour de la presse anglaise :

Il faut que la publicité inspire une grande frayeur au gouvernement, et qu'il ait beaucoup de choses à cacher. Presque toutes les nouvelles qui intéressent la France nous arrivent par l'étranger, ou par les correspondances ordinaires. Depuis que l'affaire de Taïti est engagée, le ministère a imposé à ses journaux un silence tout à fait étrange. Nous n'avons su d'abord les faits que par la publication qu'a faite $\mathrm{M}$. Pritchard dans les colonnes du Times la veille du jour où il allait se rendre avec une troupe de méthodistes, chez le premier ministre, pour réclamer contre nos officiers l'intervention de son pays. Des lettres particulières répandues dans les divers journaux ont seules rectifié les allégations du missionnaire anglais ; la presse officielle et semiofficielle de France n'a reçu aucune communication de nature à éclairer l'opinion publique.

En 1840, déjà, c'est en lisant le Morning Post, hostile à la politique de Palmerston, que le public français a pu prendre connaissance du mémorandum britannique adressé à Guizot, pour l'informer de la signature du traité de Londres ; l'opinion française était explicitement visée par le journal anglais, qui donnait une traduction du texte ${ }^{35}$.

L'État, de son côté, dénonce l'immoralité de cette prétention ; cette tendance est surtout manifeste au sud de la Manche et à droite de l'échiquier politique : l'opinion publique, représentée par une presse vénale, serait tout sauf une instance de jugement moral. Au contraire, selon le Globe de Guizot, « le journalisme a augmenté et aggravé le désordre moral des sociétés actuelles, parce qu'il les livre à cent enseignements qui ne s'accordent $\operatorname{pas}^{36}$. »

L'opinion peut d'ailleurs récuser elle-même la mauvaise publicité de la presse. En pleine affaire Pritchard, l'Adresse des ouvriers anglais aux ouvriers de France (1844) vise à contourner les mauvaises passions des journaux : « La presse des deux pays, [...] par ses vives excitations, ses bravades ou ses menaces, a cherché à rallumer la flamme de nos vieilles animosités ${ }^{37}$. » Quant aux nouveaux titres commerciaux, ils blâment l'instrumentalisation du patriotisme de l'opinion par la presse politique, y compris gouvernementale, qui fait courir un péril de guerre ${ }^{38}$.

L'exécutif français récuse jusqu'à la légitimité des débats parlementaires, en cas de crise. Le 3 août, Guizot affirme devant son chargé d'affaires à Londres son droit à soustraire les affaires étrangères à la publicité des débats : « je reviens de la Chambre des Pairs; je ne vous en dis rien. Vous verrez comme l'émotion est vive. J'ai maintenu mon droit de me taire, mes raisons de me taire, et je n'ai voulu faire aucun usage de votre dépêche de ce matin sur le discours de Sir Robert. Mais j'ai été fort poussé par les plus gros comme par les plus petits de mes adversaires ${ }^{39}$. » Guizot souhaite que son homologue britannique ne

35. Morning Post, 28 août 1840.

36. Le Globe du 31 mars 1843.

37. Adresse des ouvriers de l'Angleterre aux ouvriers de la France, Paris, imprimerie de Maulde et Renou, 1844 ; reproduit dans Révolutions du XIXe siècle, $2^{\mathrm{e}}$ série, Le mouvement ouvrier : de 1835 à 1848, 2, EDHIS, 1979.

38. La Presse, le 4 octobre 1840.

39. AN, 42 AP 7. Correspondance diplomatique privée de Guizot avec Jarnac, le 3 août 1844. 
fasse pas autrement : «Voilà notre parlement clos. Quand celui de Londres le sera-t-il ? J'en suis pressé car tant qu'il dure je suis inquiet. Il n'y a vraiment pas moyen de traiter des affaires un peu délicates à cette condition qu'à peine commencées, elles feront explosion, explosion tous les matins, explosion à Londres, explosion à Paris, mettant le feu à tout ce qui y touche ${ }^{40}$. »

L'opinion publique se revendique pourtant comme dépositaire de la moralité du pays, le mot ayant ici le sens d'honorabilité. Par une analogie entre la nation et la personne, qui mêle la modernité de la notion de nation aux valeurs de l'Ancien régime, l'opinion publique réclame que la nation insultée se comporte comme un gentilhomme défendant son honneur.

La gauche française s'inquiète, pendant l'affaire Pritchard, d'un arrangement au «mépris de la morale ${ }^{41}$. » À qui revient la charge de défendre l'honorabilité du pays ? $\mathrm{Au}$ souverain et aux institutions de l'État, plus froids et réalistes que le peuple, ou à l'opinion publique, diverse et passionnée, mais qui incarne l'idée de nation, à défaut de souveraineté populaire ? D'une certaine façon, Louis-Philippe entra dans cette dernière logique, en proposant que l'indemnité versée à Pritchard, pour solde de tout compte, fût prélevée sur sa cassette personnelle, afin d'épargner l'honneur national. Ce que Guizot refusa significativement.

Le tribunal de l'opinion, qui veille à l'honorabilité du pays, ne se borne pas à la tribune médiatique; il se donne à voir dans les lieux publics. Le banquet réformiste du 31 août 1840 s'en réjouit : «Comment [le pays ]n' aurait-il pas vu avec un vif intérêt que si le gouvernement sommeille, et s'il ne se confie encore qu'à la diplomatie qui l'a si effrontément joué, la dignité de la France, son honneur et sa force sont toujours présents à ces masses intelligentes dont le dévouement ne lui a jamais manqué ${ }^{42}$ ! »

Presse et gouvernement peuvent d'ailleurs se rejoindre : à l'annonce du traité de Londres, le Journal des débats, organe du château, est le premier à reprendre à son compte cette catégorie de l'honneur, si elle ne compte pas s'en décharger sur le peuple ou l'opinion : « on a traité sans nous, contre nous, avec une insolence que la France ne supportera pas, son honneur le lui défend ${ }^{43}$. » Paradoxe : en assumant la défense de l'honneur français, le gouvernement ne récuse pas l'existence, sinon l'utilité, du tribunal de l'opinion : c'est dans la presse, en effet, que l'exécutif prend la peine de protester de sa susceptibilité. À destination de son opinion domestique et de l'opinion étrangère.

Deuxième point, l'opinion publique est la force morale du pays ; au moins sa force se trouve-t-elle atteinte quand son image est altérée devant l'opinion internationale.

Si l'État considère généralement que les journaux divisent le pays et affaiblissent par là sa force morale, la presse de gauche répond qu'il vaut mieux une opinion avertie et turbulente, qu'apathique, dès lors que l'opinion est le ressort de la puissance publique. A Guizot, qui préfère ne pas évoquer la question tahitienne, Le Censeur de Lyon, lui conseille de « ne pas se priver au moins de l'appui moral des chambres ${ }^{44}$. » Mieux, le National l'enjoint de ne pas laisser la France souffrir la moindre offense morale sur la scène européenne, sous peine d'affaiblir le pays : «c'est pour l'Angleterre un énorme avantage

40. Ibid., le 8 août 1844 .

41. Le National du 6 septembre 1844.

42. Ibid.

43. Journal des Débats, les 29 et 30 juillet 1840.

44. Le Censeur de Lyon, le 4 août 1844. 
que d'avoir déshonoré la France, c'est-à-dire d'avoir ruiné dans l'esprit des peuples notre influence morale, notre principale force ${ }^{45}$. »

La presse britannique souligne la différence entre son opposition à la conduite immorale du gouvernement et la moralité du pays dont l'opinion publique est le garant :

Nous aimons à croire que la partie sage de la nation française ne perdra pas de vue la distinction à faire entre la politique du traité et la manière de l'exécuter. La politique du traité est britannique, et la nation en est responsable ; mais, l'exécution mesquine, perfide et provocatrice du traité appartient tout entière à lord Palmerston. C'est lui qui doit être l'objet de l'indignation, non seulement de la France, mais aussi de notre pays $^{46}$.

Le Constitutionnel de Thiers, pendant la crise orientale, s'accorde avec la gauche pour penser que l'opinion publique constitue la force morale du pays, s'il s'accorde également, avec les conservateurs, pour craindre la désunion de l'opinion. Reste qu'il s'appuie délibérément sur l'opinion pour impressionner ses interlocuteurs étrangers. À peine a-t-il appris de Guizot la nouvelle du traité de Londres, qu'il lui envoie des arguments à défendre devant Palmerston, montrant sa conception d'une puissance française fondée sur les ressorts moraux de son peuple. " $\mathrm{S}$ 'il vous répète encore que la séparation sera passagère, répondez qu'en France, les passions sont vives, [...] que le Cabinet actuel avait fait son possible pour les contenir, qu'il ne le pourra plus aujourd'hui. » De fait, pendant son entrevue avec le ministre anglais, Guizot use clairement de la menace d'une opinion publique française belliqueuse $\mathrm{e}^{47}$.

Une diplomatie morale, passée l'acception presque métaphorique que nous venons d'évoquer - les ressources non matérielles du pays -, c'est surtout l'exigence d'une politique bonne, et partant universelle. Cette exigence favorise naturellement l'émergence d'une OPI, fondée sur des valeurs partagées.

La définition par la presse du code de valeurs qui constitue une politique étrangère morale se lit le plus souvent en creux, dans la dénonciation d'un État rival. La presse française dénonce l'immoralité de la Grande-Bretagne par rapport aux usages diplomatiques et au droit des gens. Le traité de Londres de 1840 apparaît à une partie de l'opinion française comme une faute morale et pas seulement politique quand, pour les diplomates, la condamnation des procédés de Palmerston relève moins du discours moral que de celui des usages. Thiers, lui, se range résolument du côté de l'opinion. À travers le Journal des débats, il dénonce l'immoralité anglaise et en appelle à une prise en compte du peuple sur un ton presque démocratique : " nous ne sommes plus à une époque où l'on dispose du sang et de la fortune des hommes sans les consulter ${ }^{48}$ !». Devant l'ambassadeur d'Angleterre, il parle de « violence morale contre la France ${ }^{49}$. »

L'arrestation, puis l'expulsion du consul Pritchard, nourrissent une ardente polémique entre la presse des deux puissances rivales sur les usages diplomatiques. Si les Français et les Anglais en appellent symétriquement au droit des gens, les premiers ont beau jeu

45. Le 6 septembre 1844.

46. Morning Post, cité par le National du 20 septembre 1840.

47. AMAE, Affaires politiques, Angleterre, $\mathrm{n}^{\circ}$ 654, dépêche de Thiers, non datée (circa 20 juillet), réponse de Guizot le 22 juillet, f. 200'.

48. Journal des débats, le 28 juillet 1840.

49. National Archives, Foreign Office (FO), FO 27 604, le 5 octobre 1840. 
de dénoncer la possibilité, pour un consul, de se livrer à des actes séditieux. La défense de la position française - il en va de même en Angleterre - se confond avec la défense plus générale de la morale publique internationale : «Ce n'est pas notre cause seule que nous défendons, écrit le National c'est celle [...] du droit public, [...] de la justice et de la conservation des États ${ }^{50}$. »

La Grande-Bretagne, de son côté, dénonce l'immoralité de l'impérialisme et du bellicisme de l'opinion française, dans les deux crises de 1840 et 1844, plus encore que la conduite de sa diplomatie. Elle déplore l'abaissement du libéralisme chez un peuple seulement attaché à la gloire extérieure : «La rivalité avec l'Angleterre est presque le seul nerf qui demeure tendu dans l'esprit français. Tout l'amour de la liberté à l'intérieur ou à l'extérieur a disparu. Le règne des Orléans a dégoûté même les Libéraux de cela, et la gloire militaire est devenue le seul espoir et le seul but ${ }^{51}$. » La presse française répond en brocardant l'injuste prétention de l'impérialisme britannique à revendiquer une influence exclusive dans le monde ; même le très modéré Globe de Guizot réagit fermement aux protestations contre-nature de la presse britannique, à l'annonce du protectorat français sur Tahiti :

À lire les observations, moitié guerre et moitié marchandise, des journaux anglais, sur notre prise de possession des îles Marquises et sur notre protectorat des îles de la Société, on serait tenté de leur demander, encore une fois, à voir la clause du testament d'Adam qui leur adjuge cette partie du monde ${ }^{52}$.

L'exigence d'une diplomatie morale s'affirme d'une façon plus positive dans la promotion, par l'opinion, de valeurs abandonnées par son propre État au bénéfice d'une convergence ponctuelle des opinions française et britannique. Après le long cycle de guerres révolutionnaires, les forces gouvernementales en rabattent sur les prétentions vertueuses des idéalismes politiques et la presse gouvernementale répugne à user de la catégorie de moralité. Quand elle invoque la morale, c'est généralement pour l'écarter au profit d'un discours réaliste, fondé sur les intérêts. La presse gouvernementale anglaise, de son côté, ne craint pas de dénoncer la prétention de sa propre opinion quand elle réclame une diplomatie morale ; The Era, jeune hebdomadaire britannique, condamne l'immoralité des missionnaires de la London Mission Society (LMS) réunis en meeting à Exester Hall :

Tournons-nous vers une des plus répugnantes figures de notre temps; celle de la sympathie larmoyante pour les sauvages et la propagation de l'évangile par des hommes qui portent la Bible dans une main et l'épée dans l'autre [...]

Nous ne pouvons pas exprimer dans des termes plus forts l'absurdité et l'anomalie de messieurs qui au lieu d'être assis avec l'agneau jouent avec la queue du lion ${ }^{53}$.

Même opinion dans le premier journal de langue anglaise à Paris, The Galignani's Messenger, qui remplit son rôle de passerelle en dénonçant la prétention à la diplomatie morale des missionnaires anglais : « l'intolérance fanatique déployée par les orateurs de ce

50. National du 9 août 1844.

51. Morning Chronicle du 24 août 1844.

52. Le 27 mars 1843.

53. The Era, le 18 août 1844. 
meeting peut faire conjecturer l'origine des désordres et des dissensions qui se sont manifestées à Taïti ${ }^{54}$. » Le journal en vient à « blâmer très fortement le gouvernement d'avoir confié des fonctions aussi importantes que celle de consul, fonctions qui exigent avant tout un jugement calme et une sévère impartialité, à un homme comme M. Pritchard ${ }^{55}$. »

Aussi bien, la revendication d'une diplomatie morale passe-t-elle essentiellement par la presse d'opposition ou d'autres formes d'expression de l'opinion, pour défendre les valeurs abandonnées par l'État. Au premier chef, dans l'affaire Pritchard, il s'agit des valeurs de la religion. En France, les journaux protestants dénoncent la diplomatie immorale de l'État catholique, ulcérés que le huguenot François Guizot prône la défense d'un catholicisme d'État. En Angleterre, c'est le non-conformisme qui proteste contre la politique indifférente de l'État anglican - quoique la presse gouvernementale et commerciale soit majoritairement pleine de suspicion pour l'intransigeance des missionnaires. Mais il est aussi question de la tartuferie religieuse des décideurs, notamment dans les milieux réformistes, volontiers libres penseurs. Les ouvriers anglais dénoncent l'immoralité de leur gouvernement et des missionnaires de la LMS, qui prétendent incarner la religion, et prônent la guerre au lieu de défendre la paix ${ }^{56}$. Les pamphlétaires libéraux, en France, moquent l'hypocrite morale chrétienne de Guizot, avec une pointe d'anti-protestantisme, sur l'air de Cadet Roussel :
Pour mal il rend toujours le bien
(ceci est d'un très-bon chrétien).
Il est surtout très-pacifique
Pour toute insulte britannique
Oui, oui,
Certainement
Lord Guizot est un bon enfant $5^{57}$

Il est question, enfin, du droit des peuples à disposer d'eux-mêmes. En confondant quelque peu la défense du protestantisme avec le principe de nationalité, l'Espérance dénonce la prétention de Dupetit-Thouars à annexer Tahiti au mépris des dispositions du peuple tahitien : « on court le risque de fouler aux pieds les premières règles de l'équité internationale et de la probité politique ». La défense du droit des Tahitiens à disposer d'eux-mêmes entre en résonance avec une revendication beaucoup plus courante, en faveur des Polonais, Belges, Italiens ou Grecs que l'État français abandonne aux Puissances despotiques; morale universelle régulièrement défendue dans la presse patriote et aux banquets réformistes, depuis plus d'une décennie.

La promotion d'une politique vertueuse a favorisé l'émergence d'une OPI dépassant les intérêts nationaux au profit de principes universels. En défendant la paix entre nations civilisées d'égale dignité, contre le bellicisme d'État, les opinions publiques françaises

54. Le journal remplit le même rôle de passeur, sur le plan technique et commercial, en introduisant dans la presse française les avancées anglaises. Diana Cooper-Richet, «Les imprimés en langue anglaise en France au XIX ${ }^{\mathrm{e}}$ siècle ; rayonnement intellectuel, circulation et modes de pénétration », Jacques Michon et Jean-Yves Mollier (dir.), Les mutations du livre et de l'édition dans le monde du XVIII siècle à l'an 2000, L'Harmattan, 2001, p. 128 et $s q$.

55. Cité par L'Illustration du 24 août 1844.

56. Adresse des ouvriers de l'Angleterre, op. cit.

57. Antoine-François Lallemant, dit Francique, Lord Guizot, chanson élégiaque par M. MM. Francisque $L^{* * *}$, Paris, impr. de Guillois, 1846. 
et anglaises ont participé à l'invention de la notion d'arbitrage : le tribunal informel de l'opinion préfigure, en somme, la judiciarisation de la société internationale. Mais l'opinion publique a aussi été le moyen de revendiquer une démocratisation des nations et de leurs relations.

\section{L'arbitrage de l'opinion et l'idéal républicain d'une diplomatie des peuples, facteurs de paix ou de guerre ?}

La notion d'arbitrage demeurait largement inaboutie dans les années 1840. Dans la presse, l'évocation d'un arbitrage par une OPI est rare et manque de conviction. Le plus souvent, nos crises sont l'occasion d'une segmentation des opinions par logiques nationales, dressées les unes contre les autres, sans parler des fractures domestiques, selon les principes religieux, politiques ou sociaux déjà évoquées. De loin en loin, le souci d'alerter une hypothétique opinion publique européenne est formulé par un journal. C'est ce que souhaite le National du 18 février 1844, à l'annonce de l'annexion de Tahiti par Dupetit-Thouars : « Ni le Messager ni le Moniteur parisien n'en parlent ce soir et cela est fâcheux. Il importe beaucoup, en effet, d'édifier, dès le premier moment, l'opinion publique en Europe. [...] il faut exposer, sans délai, l'affaire dans tous ses détails au jugement du public européen ». Selon la même logique transnationale, suivant cette fois une solidarité religieuse, la presse huguenote alerte le protestantisme européen, ce qui la met bientôt en mesure de publier « une adresse [...] signée par les quatre sociétés des Missions évangéliques qui existent en Allemagne ${ }^{58}$. »

L'idée de recourir à un tribunal de l'opinion, élargi à un espace public européen, existe à l'état de prolégomènes ; mais ce principe dissimule souvent des intérêts de puissance. Il n'obéit à aucune procédure institutionnalisée, ni ne convoque seulement une conception claire de cette opinion, relevant souvent d'une simple logique d'invocation, pour légitimer un fait accompli. Suivant cette logique, une partie de l'opinion publique britannique, pour contester le protectorat français sur Tahiti, qui menace l'évangélisation protestante, en appelle à l'arbitrage moral d'une très inconsistante OPI : « La nation française encoure la censure des honnêtes hommes [good men] de toutes les nations ${ }^{59}$. »Pour juger ce que les Britanniques estiment une violation du droit des gens, l'opinion européenne devient une sorte de succédané très informel à un tribunal international qui n'existe pas, cinquante-cinq ans avant l'institution de la Cour permanente d'arbitrage. Au début de l'affaire Pritchard, The Patriot entend la cause en proposant de renverser les rôles, pour en appeler à un jugement équitable : «Son arrestation et son bannissement sont en contravention flagrante avec la loi internationale, et en changeant les rôles, ce serait là assurément, aux yeux de nos plus belliqueux voisins, un casus belli ${ }^{60}$. » L'invocation d'une opinion publique européenne au bénéfice de ses thèses permet aussi à la presse d'opposition de critiquer la politique gouvernementale sans manquer à la solidarité nationale. Dans la crise de 1840 , le Times, journal tory par excellence, attaque la politique agressive de Palmerston à l'égard de la France. Il exige une meilleure publicité de sa politique : «le gouvernement doit soumettre aux délibérations du parlement ou faire connaître au pays les moyens qu'il compte employer, et certainement si lord Palmerston avait conclu un traité qu'il serait plus désastreux d'exécuter que de rompre, [...] la nation britannique n'hésiterait pas à le briser ». Mais il balance aussitôt son audace démocratique et atténue la portée de sa critique, pour étayer la position britannique sur le fond, en prenant en otage la fameuse OPI : «Nous

58. L'Espérance du 9 août 1844.

59. The Standard, le 27 février 1844.

60. The Patriot, cité dans le Constitutionnel du 3 août 1844. 
approuvons, avec l'immense majorité du public européen, les objets que l'on s'est proposé de réaliser par le quadruple traité61 ${ }^{61}$ »

Le fait que cette notion d'arbitrage par une opinion internationale soit rarement invoquée de façon explicite par la presse ne signifie pas qu'elle n'ait pas opéré de facto, produisant ses effets iréniques serait-ce que dans la seule sphère commune aux opinions française et britannique. La connaissance réciproque de l'opinion adverse a certainement nourri l'effervescence dans chaque espace public, par effet de miroir ; mais il a contribué également à l'information et la prise de conscience des périls de guerre des deux côtés de la Manche.

La presse de chaque pays traduit abondamment celle de l'adversaire, pour s'en agacer, mais aussi pour prendre la mesure du péril ou rassurer le public. Quand le National traduit en 1840 le Morning-Chronicle de Palmerston, «Que les Français ne regardent pas une guerre avec la Grande-Bretagne comme une bagatelle. Nous serions maîtres de la mer », c'est pour s'en indigner, et répondre sur un ton bravache ; mais c'est aussi l'occasion de réfléchir : «En cas de faute ou d'insuccès, les avertissements, comme on le voit, ne nous aurons pas manqué62 ${ }^{2}$ » Les journaux britanniques analysent soigneusement la presse française pour conclure au pacifisme foncier de l'opinion, par-delà les gesticulations de quelques titres. C'est ce dont veulent convaincre les missionnaires anglais quand ils prennent la parole au grand meeting de la LMS, à Exester Hall, le 14 août 1844 : « Le cri de la guerre vient d'une portion corrompue et vénale de la presse française, une presse qui est complètement enchaînée (fettered) et sous le contrôle de la cour, et qui n'exprime pas les sentiments du peuple ${ }^{63}$. » On voit à cette occasion resurgir la distinction entre l'opinion, faite de représentations volatiles et diverses, et le peuple, stable et réel. C'était déjà tout l'effort de pédagogie de Palmerston et de son Morning Chronicle en 1840 et, plus généralement, de toute la presse whig ministérielle :

Je ne crois pas qu'il y aura la guerre au sujet de la question Syrienne. [...] L'ardeur au combat que l'on trouve des cafés parisiens n'affecte que rarement et sous une forme atténuée la province. La presse parisienne, de plus, ne reflète que l'opinion des cafés de Paris, pas de l'ensemble de la nation. Il y a un contraste bien marqué entre le journalisme français et l'anglais à cet égard. Il est important de le noter. La circulation d'un journal national, en France, est insignifiante en province ; la capitale l'absorbe presque entièrement. Ici, les journaux de Londres, et les opinions qui y sont exprimées, sont couramment lues dans tout le pays. Il est loin d'en être de même en France : en dehors des grandes villes, un journal parisien est presque inconnu, et les Feuilles d'Annonces, qui constituent $90 \%$ de la presse provinciale en France, ne discutent ni retranscrivent les opinions de la presse nationale. D'où la vanité de prendre la presse parisienne comme un indicateur de l'état d'esprit national. L'une des peurs dominant le peuple, en France, c'est la conscription ${ }^{64}$.

Même la presse conservatrice hostile à Palmerston, pendant la crise d'Orient, fait cet effort de décodage des pratiques médiatiques françaises. La meilleure connaissance

61. Cité dans le National du 25 août 1840.

62. Le National du 6 août 1840.

63. Morning Chronicle du 15 août 1844.

64. Le 18 août 1840, sous la forme d'un courrier de lecteur, qu'il est évidemment tentant de prêter à la rédaction. Cela continue de la même façon dans la deuxième quinzaine d'août et au début du mois de septembre. On retrouve les mêmes propos rassurants tout au long de la crise dans le Globe par exemple. 
réciproque participe de l'émergence, sinon d'un espace public, du moins d'espaces publics sécants, permettant d'apprivoiser la culture médiatique adverse. Lorsque Thiers alimente la passion belliqueuse françaises, en annonçant la construction de fortifications, le Morning Advertiser rassure son lectorat : «Les écrivaillons [hacks]ministériels, comme ceux du Constitutionnel, du Courrier français du Siècle, du Temps et du Messager défendent la mesure, comme ils s'y seraient opposés si on leur avait demandé de les combattre ${ }^{65}$. »Et le Standard conservateur de Peel ne rassure pas moins l'opinion britannique que la presse de Palmerston en affirmant : « Les armements ordonnés par $\mathrm{M}$. Thiers peuvent faire croire à la guerre au premier abord ; mais, avec un peu de réflexion, on voit que c'est plutôt une démonstration que des préparatifs sérieux d'hostilité. [...] Aucune guerre n'aura lieu ${ }^{66}$. »

Dans quelle mesure l'emballement médiatique a-t-il permis d'apurer le prurit patriotique, dans chacune des crises franco-anglaises ? Mais l'exaspération du sentiment national, dans son expression même, qui était aussi un jeu, dans l'espèce de volupté à affirmer son bon droit, dans la bonne peur consistant à évaluer les périls, visait certainement plus à préserver la paix qu'à préparer la guerre. À éviter la guerre en la cantonnant à la sphère du verbe. Même l'ultranationaliste et apparemment belliqueux John Bull prônait la fermeté comme meilleur moyen d'éviter la guerre ${ }^{67}$. Sans parler de la presse commerciale ou conservatrice, en France, qui dénonça le péril de guerre, en 1840 comme en 1844, les titres les plus patriotiques, comme le National ou le Capitale ou le Siècle, dans une moindre mesure, ne font pas autrement que John Bull : leurs vociférations sont conçues pour défendre la paix sans perdre l'honneur. Lorsque le bonapartiste Capitole s'écrie, le 28 juillet 1840 : « Nous n'apercevons donc que l'alternative de se fâcher ou de s'humilier à deux genoux », il s'agit bien de se fâcher pour faire peur, et non pas faire la guerre. C'est en faisant bien voir au cabinet de Londres la résolution française à faire la guerre que la guerre ne sera pas nécessaire : «Il a méconnu la France, lorsqu'il a pensé que toutes ses querelles de parti et toutes ses dissensions intérieures ne s'effaceraient pas momentanément devant une question d'honneur national posée d'une façon aussi nette et aussi précise ». Le Siècle résume ainsi la nécessité de parler haut pour préserver la paix : « le ministère, tout en négociant, se tient sur ses gardes et se prépare à soutenir la guerre avec vigueur. Nous l'approuvons de ne vouloir point la provoquer ; mais l'activité de ses préparatifs, que nous ne saurions mettre en doute, est pour nous une garantie beaucoup plus sûre que les espérances qu'il apparaît fonder quelques fois encore dans la sagesse et les dispositions pacifiques des gouvernements de l'Europe ${ }^{68}$. »

Aussi bien, quand Lamartine écrit dans la Presse : " il n'y a rien de si impopulaire que la paix dans un pays dont la glorieuse faiblesse est la guerre. C'est le devoir, cependant, de tout homme de le dire », il remplit ce devoir sans excès de masochisme : il connaît le pacifisme foncier de sa clientèle politique, il sait la popularité que son propos lui vaudra dans les milieux conservateurs et outre-Manche, où son article est abondamment cité ${ }^{69}$. La paix demeurait l'horizon partagé du bien commun, pour une immense majorité de l'opinion, des deux côtés de la Manche.

65. Morning Advertiser, le 14 septembre 1840.

66. Cité par le National du 6 août.

67. John Bull, le 4 août 1844, se félicite du ton exigeant des ministres britanniques : « Nous aimons voir une saine résolution résolument exprimée. Cela a un bon effet, et permet généralement d'éviter des soucis ultérieurs ». Le Morning Chronicle exige la plus grande fermeté pour la même raison : éviter la guerre en montrant qu'on ne la craint pas (dans la première de ses éditions du 7 août par exemple).

68. Le Siècle du 30 août 1840.

69. Article repris et commenté notamment par le Morning Advertiser du 28 août 1840. 
L'invocation, rare et confuse, d'une OPI arbitre des conflits internationaux, peu probante étant donné l'étroitesse de l'espace public européen dans les années 1840 ; la pratique, plus efficiente, de la prise en compte réciproque du désir de justice et d'honorabilité dans les rapports internationaux : tout cela ne doit pas être confondu avec l'appel à une diplomatie des peuples qui, pour relever également d'une logique internationale, récuse la notion d'opinion.

Une diplomatie des peuples pour une diplomatie morale : cette notion apparaît négativement, sur le plan international, comme le refus des peuples d'être opposés entre eux, posture consubstantielle au refus d'être privé, pour chacun d'entre eux, de la maitrise des questions de politique étrangère. Pour Le Constitutionnel, qui exprime en 1844 les vues de l'opposition de la gauche de gouvernement, l'échec de l'entente cordiale entre la France et l'Angleterre ne procède pas d'un fatal bellicisme des opinions, mais du procédé et du rythme d'un rapprochement opéré par les gouvernements, sans consulter les peuples ${ }^{70}$. La presse d'opposition au régime, républicaine, est plus explicite, en liant la revendication d'une prise en compte de l'opinion publique avec celle d'une réforme du suffrage :

Si les alliances fondées sur le vœu des peuples sont plus solides que les autres, il est utile, il est nécessaire que le vœu du peuple se puisse manifester sur les questions de paix, de guerre et d'alliance. [...] il est utile, nécessaire, indispensable de modifier la Charte, et d'enlever au pouvoir royal le droit exclusif qu'il possède aujourd'hui de faire les traités de paix et d'alliance.

Mais ce droit, à qui l'attribuer [...] ? A la réforme radicale de notre système électif, au suffrage universel ${ }^{71}$.

Les républicains réclament pour le peuple le pouvoir de dire quelle est la bonne politique et d'en être l'acteur ; il ne s'agit plus de recourir à l'arbitrage d'une opinion publique transnationale mais de réinvestir les lieux publics, pour décider collectivement de la politique étrangère. Cette revendication se confond avec la promotion d'une politique morale, contre la diplomatie des rois qui est injuste, ne serait-ce que parce qu'elle n'est que celle des rois, comme l'expliquent les orateurs du banquet de Chatillon :

Pourquoi le peuple ne serait-il pas appelé à nommer ses représentants et ses administrateurs ? A-t-il moins de moralité, d'indépendance et de jugement que la parcelle privilégiée dont le fol orgueil va jusqu'à la prétention de comprendre les intérêts du peuple mieux que le peuple lui-même?

Et de souhaiter le « ralliement des peuples contre la collation des rois ${ }^{72}$. »

L'appel à une diplomatie des peuples se formalise surtout à travers les premiers linéaments d'une conscience de classe refusant l'immoralité des politiques gouvernementales au nom d'intérêts communs aux ouvriers, qui entendent fonder un ordre international sur la fraternité des travailleurs.

La conscience de classe, chez les ouvriers anglais et français, la naissance d'une sphère publique plébéienne, dotée d'une culture spécifique, avec ses lieux propres, café, tavernes, Églises dissidentes en Angleterre, étaient choses trop fragiles pour spéculer sur

70. Le Constitutionnel du 3 août 1844.

71. Le National du 15 août 1840.

72. Le National, ${ }^{\mathrm{er}}$ septembre 1840. 
la capacité d'action du pacifisme ouvrier transnational des années $1840^{73}$. Mais n'est-il pas remarquable, qu'au plus chaud de l'affaire Pritchard, la presse républicaine, en France, ait souhaité, sans s'illusionner pour autant, l'unité des classes laborieuses pour éviter une guerre préparée par les gouvernements ? Le National observe : « toute la partie de la presse [anglaise ]qui s'adresse aux hautes classes et à la bourgeoisie attise de toutes ses forces les vieilles haines nationales. La presse qui s'adresse au peuple est beaucoup plus calme ». En 1840, déjà, pour blâmer la diplomatie des chancelleries, qui ne tenait pas compte du vœu des peuples, le National prédisait le triomphe d'une nouvelle organisation européenne du fait des progrès de l'idée démocratique et des solidarités de classe transnationales :

Les intérêts créent des distances, entre les nations : les idées les effacent. Plus nous avançons, plus nos idées deviennent envahissantes ; on a beau s'armer contre elles de lois de police et de tarifs de douanes, elles pénètrent, malgré tous les efforts ; elles gagnent ceux-là même qui les repoussent; et comme elles sapent dans sa base l'état social de l'Europe, elles sont une menace perpétuelle contre les constitutions aristocratiques et despotiques du continent ${ }^{74}$.

De fait, au cœur de l'affaire Pritchard, les ouvriers anglais vont donner un témoignage de leur solidarité de classe en faveur de la paix, contre l'immoralité des gouvernements, en publiant, à l'initiative de William Lovett une « Adresse des ouvriers de l'Angleterre aux ouvriers de la France », saturée d'invocations morales :

Frères, nous croyons pouvoir vous donner ce titre, car si l'Océan nous sépare, la justice de nos principes et la conformité de nos intérêts doivent nous réunir ; [...] la charité chrétienne doit nous rappeler à notre devoir, et nous faire mépriser ceux qui nous engagent à briser les liens de la morale, et à plonger nos deux pays dans une guerre injuste et dévastatrice. [...]

La guerre est, selon nous, le plus grand des maux, soit à cause des effets démoralisants qu'elle produit, soit parce qu'elle retarde les progrès intellectuels, moraux et physiques du genre humain.

Un autre leader chartiste, Feargus O'Connor, plaide dans The Northern Star and Leeds General Advertiser du 17 août 1844, pour que les peuples dépassent les «factions » ou « groupes d'opinion », qui produisent des « sentiments égoïstes ». Il prend à contrecourant les discours patriotiques en refusant toute guerre qui prolongerait « la tyrannie domestique ».

En bons héritiers de Kant et de son opuscule fondant la paix perpétuelle sur la généralisation d'un régime républicain, les acteurs de la gauche républicaine française souhaitent fonder la paix sur une démocratie universelle, permettant une diplomatie des peuples. Telle est la conclusion du National rapportant le banquet de Chatillon : " [Le peuple ][et non l'opinion ]a les traditions de gloire ; il a de plus le sentiment de fraternité qui doit unir entre elles toutes les nations. Il veut la grandeur du pays ; mais cette grandeur n'est pas pour lui l'esprit turbulent des conquêtes ; cette grandeur, il la fonde sur une organisation

73. Edward P. Thompson, La formation de la classe ouvrière anglaise, Gallimard, Seuil, 1988 (éd. originale 1963).

74. Le National des 30-31 juillet 1840. 
à laquelle l'égalité préside à l'intérieur, que l'alliance démocratique assure et garantit au dehors... ${ }^{75}$.»

Cette diplomatie des peuples, la presse britannique la craignait, si la France devait user du levier démocratique pour lever les peuples contre l'Europe despotique ; mais elle ne croyait pas les dirigeants français assez républicains pour cela : «Louis-Philippe et M. Thiers oseraient-ils se mettre à la tête d'un mouvement de ce genre ? Qu'ils évoquent l'esprit électrique de la France démocratique, et ils seront peut-être les premières victimes. Nous croyons en conséquence qu'une pareille éventualité n'est pas à redouter ${ }^{76}$. » De sorte que l'opinion libérale, en Grande-Bretagne, pouvait escompter que l'esprit démocratique français permettrait aux deux nations les plus avancées d'Europe de guider et arbitrer de concert les autres Puissances, au lieu de se faire le ferment d'une révolution visant à fédérer une Europe républicaine. C'est dans cet esprit que poursuivait le journaliste du Morning Adverstiser :

Les intérêts les plus chers de l'humanité pourraient être compromis par une collision entre la France et l'Angleterre. Ces deux nations sont heureusement à la tête de la civilisation et d'une liberté éclairée, et fasse le ciel qu'elles occupent longtemps cette belle position, en paix l'une avec l'autre, protégeant les opprimés et améliorant, avec leurs propres intérêts, les intérêts généraux de la race humaine.

Pour conclure, il va de soi que les gouvernements français et britannique n'entendaient pas se plier à l'arbitrage de l'opinion publique dans les crises des années 1840 ; ils ont moins encore favorisé l'avènement d'une démocratie domestique pour refonder l'ordre européen sur l'alliance universelle et pacifique des démocraties. Reste que les questions étrangères ont été prises en compte par un public toujours plus large, dans ces deux pays en voie de démocratisation ; un public toujours plus impatient de faire valoir son opinion. Cette exigence de publicité marque, en soi, une évolution des pratiques diplomatiques. En se saisissant des questions de politique étrangère, dans toute la diversité de ses intérêts et de ses conceptions, l'opinion publique a-t-elle augmenté le péril de guerre, comme ont pu le croire les dirigeants de l'époque?

Sur le vif, comme a posteriori, Aberdeen et Guizot ont donné le sentiment d'avoir dépassé les passions de l'opinion pour traiter froidement l'émotion causée par l'affaire Pritchard $^{77}$. Si Palmerston et Thiers ont cyniquement joué des passions nationales, en 1840, laissant voir qu'ils utilisaient cette nouvelle composante de la puissance, au risque de se faire dévorer par elle, on aurait tort de croire que leurs successeurs ont usé avec plus de sagesse de l'opinion, pour lui imposer une paix dont elle ne voulait pas. Certes, les dirigeants ont souvent cherché à modérer leur opinion : lorsque l'ambassadeur de France annonce à Aberdeen le protectorat français sur Tahiti, il lui demande d'épargner aux missionnaires anglais des «mesures vexatoires et arbitraires qui auraient ici un retentissement fâcheux, et auxquelles l'opinion publique ne nous permettrait pas de rester indifférents ${ }^{78}$. » Mais ils soufflaient le froid après le chaud, et voulaient calmer une opinion qu'ils avaient délibérément excitée par des aventures qu'elle ne souhaitait pas. Une analyse fine de la crise tahitienne, qui court de 1842 à 1844, donne raison à Rémusat, quand il reproche à « Guizot de s'être jeté sans de solides raisons dans le guêpier de l'affaire

75. Le National du $1^{\mathrm{er}}$ septembre 1840.

76. Morning Advertiser cité dans le National du 4 août 1840.

77. C'est le jugement de Laurent Theis, François Guizot, Fayard 2008.

78. AMAE, Affaires politiques, Angleterre, 661, f. 43, Saint-Aulaire à Guizot, le 21 mars 1843. 
de Tahiti ${ }^{79}$. » Il faudrait préciser : pour des raisons de prestige et de politique intérieure plutôt que d'intérêt national. Guizot a activé une sensibilité et créé une susceptibilité qui n'existait pas autour des enjeux océaniens et réveillé le patriotisme d'une opinion publique apaisée après les événements de 1840. Si l'opinion française devient largement belliciste, à l'été 1844, unifiée jusqu'aux protestants dans l'hostilité à la Grande-Bretagne, c'est que les courants les plus profonds du sentiment national ont été réactivés artificiellement par Guizot $^{80}$. C'est à l'inconséquence de Guizot, qui avait lancé une aventure coloniale non désirée, pour reculer ensuite devant les résistances des missionnaires britanniques, tout en refusant d'informer son opinion sur les développements de l'affaire, qu'il faut imputer le bellicisme de l'opinion française à l'été 1844. Une opinion excitée plutôt qu'éclairée. Telle fut l'analyse de l'Examiner, sur le vif. L'avis du journal britannique était conditionné par son présupposé libéral et même radical, mais il sonne singulièrement juste, à distance, quand il explique le désir de gloire extérieure du public français par le besoin d'une compensation au déficit de liberté intérieure :

Dans la syncope où a plongé la vie politique intérieure en France, la seule question qui offre vie et force est la politique étrangère ; et par conséquent il faut en faire quelque chose. Les Français sont si excitables ; la clameur du public, non pas pour réclamer la guerre, mais contre Guizot, est si considérable, que si lui et la paix se confondent encore dans l'esprit du public, ils pourraient se perdre ensemble ${ }^{81}$.

Avant Guizot, Thiers et Palmerston avaient joué avec l'opinion publique pour soigner leur popularité. L'opinion française n'était pas dupe de cette instrumentalisation ; la presse de tout bord la dénonçait, soit pour craindre, soit pour souhaiter que l'opinion publique libère un peuple ainsi stimulé. La presse anglaise blâmait les deux gouvernements de chercher à instrumentaliser les passions publiques et le journaliste du Morning Post manifesta une cruelle auto-dérision pour sa profession, en appelant Thiers et Palmerston, au cœur de la crise de 1840, « à écrire dans leurs journaux respectifs des choses brillantes contre l'empire de l'autre » plutôt que d'exciter leurs opinions respectives depuis leur cabinet ministériel $^{82}$. Aussi bien, le bellicisme d'État, purement verbal et déceptif, n'a pas pesé pour rien dans le rejet de la monarchie de Juillet, en 1848. Il n'est qu'à voir la place tenue par l'affaire Pritchard, par-delà ses effets quotidiens dans la presse, dans la littérature contemporaine, chez Hugo et Flaubert notamment ${ }^{83}$.

Du côté britannique, l'usage de l'opinion par les décideurs dépendait des tendances politiques. Pour Palmerston, le sentiment national, la passion des affaires publiques étaient de bonnes choses en soi, qu'il était loisible de flatter et surtout d'utiliser, pour défendre les intérêts britanniques. Les conservateurs, comme Aberdeen, craignaient de se laisser entraîner par leur opinion ; mais ils en tenaient compte. Les uns et les autres ne pouvaient pas douter du pacifisme foncier de l'opinion publique. Son agressivité, mis à part les milieux piétistes, procédait avant tout d'une crainte obsidionale, face à un impérialisme français mal évalué ; elle demeurait si peu belliciste que les whigs, constituant l'opposition lorsque Aberdeen dut se dépêtrer de l'affaire tahitienne, incitèrent leur champion à la modération,

79. Charles de Rémusat, op. cit., p. 54.

80. Je me permets de renvoyer à mon étude spécifique de l'opinion française face à l'aventure tahitienne, « "Ici l'on danse" : Tahiti et l'opinion publique française sous la Monarchie de Juillet », Hermès, n 65, avril 2013.

81. The Political Examiner, le 17 août 1844.

82. The Morning Post du 17 septembre 1844.

83. Renaud Meltz, «L'affaire Pritchard », art. cit. 
afin que leur parti ne devienne pas celui de la guerre. Au lendemain de l'affaire, Macaulay écrivit à Palmerston : «Personne ne sait mieux que vous, $\mathrm{j}$ 'en suis sûr, que de toutes les imputations qu'on peut jeter à des hommes politiques, celle d'être un parti de guerre, est dans la situation présente de l'opinion publique, la plus préjudiciable ${ }^{84}$.»

UNIVERSITÉ DE POLYNÉSIE FRANÇAISE

84. Roger Buellen, Palmerston, Guizot and the Collapse of the Entente Cordiale, London, Athlone, 1974, p. 43, lettre du 9 janvier 1845 . 